\title{
Inequalities for eigenvalues of matrices
}

\author{
Xiaozeng $\mathrm{Xu}^{1,2^{*}}$ and Chuanjiang $\mathrm{He}^{1}$
}

\section{"Correspondence:} cquxuxz@163.com

${ }^{1}$ College of Mathematics and Statistics, Chongqing University, Chongqing, 401331, P.R. China 2School of Mathematics and Statistics, Chongqing University of Technology, Chongqing, 400054, P.R. China

\begin{abstract}
The purpose of the paper is to present some inequalities for eigenvalues of positive semidefinite matrices.
\end{abstract}

MSC: $15 \mathrm{~A} 18 ; 15 \mathrm{~A} 60$

Keywords: singular values; eigenvalues; unitarily invariant norm

\section{Introduction}

Throughout this paper, $M_{n}$ denotes the space of $n \times n$ complex matrices and $H_{n}$ denotes the set of all Hermitian matrices in $M_{n}$. Let $A, B \in H_{n}$; the order relation $A \geq B$ means, as usual, that $A-B$ is positive semidefinite. We always denote the singular values of $A$ by $s_{1}(A) \geq \cdots \geq s_{n}(A)$. If $A$ has real eigenvalues, we label them as $\lambda_{1}(A) \geq \cdots \geq \lambda_{n}(A)$. Let $\|\cdot\|$ denote any unitarily invariant norm on $M_{n}$. We denote by $|A|$ the absolute value operator of $A$, that is, $|A|=\left(A^{*} A\right)^{\frac{1}{2}}$, where $A^{*}$ is the adjoint operator of $A$.

For positive real number $a, b$, the arithmetic-geometric mean inequality says that

$$
\sqrt{a b} \leq \frac{a+b}{2}
$$

It is equivalent to

$$
(a b)^{m} \leq\left(\frac{a+b}{2}\right)^{2 m}, \quad m=1,2, \ldots
$$

Let $A, B \in M_{n}$ be positive semidefinite. Bhatia and Kittaneh [1] proved that for all $m=$ $1,2, \ldots$,

$$
\lambda_{j}\left((A B)^{m}\right) \leq \lambda_{j}\left(\frac{A+B}{2}\right)^{2 m} .
$$

This is a matrix version of (1.1). For more information on matrix versions of the arithmeticgeometric mean inequality, the reader is referred to [1-11] and the references therein.

It is easy to see that the arithmetic-geometric mean inequality is also equivalent to

$$
\left(a^{3 / 4} b^{3 / 4}\right)^{2 / 3} \leq \frac{a+b}{2} .
$$

As pointed out in [10, p.198], although the arithmetic-geometric mean inequalities can be written in different ways and each of them may be obtained from the other, the matrix versions suggested by them are different.

(c) 2013 Xu and He; licensee Springer. This is an Open Access article distributed under the terms of the Creative Commons Attribution License (http://creativecommons.org/licenses/by/2.0), which permits unrestricted use, distribution, and reproduction in any medium, provided the original work is properly cited. 
In this note, we obtain a refinement of (1.2) and a log-majorization inequality for eigenvalues. As an application of our result, we give a matrix version of (1.3).

\section{Main results}

We begin this section with the following lemma, which is a question posed by Bhatia and Kittaneh [1] (see also [8, 10]) and settled in the affirmative by Drury in [2].

Lemma 2.1 Let $A, B \in M_{n}$ be positive semidefinite. Then

$$
s_{j}(A B) \leq s_{j}\left(\frac{A+B}{2}\right)^{2} .
$$

As a consequence of Lemma 2.1, we have

$$
\left\||A B|^{1 / 2}\right\| \leq \frac{1}{2}\|A+B\|
$$

It is a matrix version of the arithmetic-geometric mean inequality. By properties of the matrix square function, we know that this last inequality is stronger than the assertion

$$
\|A B\| \leq\left\|\left(\frac{A+B}{2}\right)^{2}\right\|
$$

which is due to Bhatia and Kittaneh [1] and is also a matrix version of (1.1).

Theorem 2.1 Let $A, B \in M_{n}$ be positive semidefinite. Then for all $m=1,2, \ldots$,

$$
\lambda_{j}\left((A B)^{m}\right) \leq \lambda_{j}\left(\frac{A+B+A^{1 / 2} B^{1 / 2}+B^{1 / 2} A^{1 / 2}}{4}\right)^{2 m} .
$$

Proof By Lemma 2.1, we have

$$
\begin{aligned}
\lambda_{j}\left(\left(A^{2} B^{2}\right)^{m}\right) & =\left(\lambda_{j}\left(A^{2} B^{2}\right)\right)^{m} \\
& =\left(\lambda_{j}\left(A B^{2} A\right)\right)^{m} \\
& =\left(s_{j}(A B)\right)^{2 m} \\
& \leq s_{j}\left(\frac{A+B}{2}\right)^{4 m} \\
& =\lambda_{j}\left(\frac{A+B}{2}\right)^{4 m} .
\end{aligned}
$$

Replacing $A, B$ by $A^{1 / 2}, B^{1 / 2}$ in (2.3), we have

$$
\lambda_{j}\left((A B)^{m}\right) \leq \lambda_{j}\left(\frac{A+B+A^{1 / 2} B^{1 / 2}+B^{1 / 2} A^{1 / 2}}{4}\right)^{2 m} .
$$

This completes the proof. 
Remark 2.1 Let $A, B \in M_{n}$ be positive semidefinite. Note that

$$
0 \leq \frac{\left(A^{1 / 2}-B^{1 / 2}\right)^{2}}{2}=\frac{A+B}{2}-\frac{A+B+A^{1 / 2} B^{1 / 2}+B^{1 / 2} A^{1 / 2}}{4} .
$$

Therefore, the inequality (2.2) is a refinement of the inequality (1.2).

Remark 2.2 For $m=1$, by (1.2), we have

$$
\lambda_{j}(A B) \leq \lambda_{j}\left(\frac{A+B}{2}\right)^{2} .
$$

For $m=1$, by (2.2), we have

$$
\lambda_{j}\left(A^{2} B^{2}\right) \leq \lambda_{j}\left(\frac{A+B}{2}\right)^{4} .
$$

In view of the inequalities (2.4) and (2.5), one may ask whether it is true that

$$
\lambda_{j}\left(A^{m} B^{m}\right) \leq \lambda_{j}\left(\frac{A+B}{2}\right)^{2 m}
$$

for all $m=1,2, \ldots$ The answer is no. For $m=3$, the inequality (2.6) is refuted by the following example:

$$
A=\left[\begin{array}{ll}
5 & -1 \\
-1 & 9
\end{array}\right], \quad B=\left[\begin{array}{ll}
6 & -4 \\
-4 & 5
\end{array}\right] .
$$

Theorem 2.2 Let $A, B \in M_{n}$ be positive semidefinite. Then

$$
\prod_{j=1}^{k}\left|\lambda_{j}\left(A\left(\frac{A^{v} B^{1-v}+A^{1-v} B^{v}}{2}\right) B\right)\right| \leq \prod_{j=1}^{k} \lambda_{j}\left(\frac{A+B}{2}\right)^{3} .
$$

Proof By Weyl's inequality, Horn's inequality and Lemma 2.1, we have

$$
\begin{aligned}
\prod_{j=1}^{k}\left|\lambda_{j}(A X B)\right| & =\prod_{j=1}^{k}\left|\lambda_{j}(X A B)\right| \\
& \leq \prod_{j=1}^{k} s_{j}(X A B) \\
& \leq \prod_{j=1}^{k} s_{j}(X) s_{j}(A B) \\
& \leq \prod_{j=1}^{k} s_{j}(X) \prod_{j=1}^{k} s_{j}\left(\frac{A+B}{2}\right)^{2} .
\end{aligned}
$$

Putting

$$
X=\frac{A^{v} B^{1-v}+A^{1-v} B^{v}}{2}, \quad 0 \leq v \leq 1,
$$


in (2.7) gives

$$
\prod_{j=1}^{k}\left|\lambda_{j}\left(A\left(\frac{A^{v} B^{1-v}+A^{1-v} B^{v}}{2}\right) B\right)\right| \leq \prod_{j=1}^{k} s_{j}\left(\frac{A^{v} B^{1-v}+A^{1-v} B^{v}}{2}\right) \prod_{j=1}^{k} s_{j}\left(\frac{A+B}{2}\right)^{2} .
$$

In response to a conjecture by Zhan [11], Audenaert [3] proved that if $0 \leq v \leq 1$, then

$$
s_{j}\left(\frac{A^{v} B^{1-v}+A^{1-v} B^{v}}{2}\right) \leq s_{j}\left(\frac{A+B}{2}\right) .
$$

The special case where $v=\frac{1}{2}$ was obtained earlier in $[6,12]$ and the special case where $v=\frac{1}{4}$ was obtained earlier in [13]. It follows from (2.8) and (2.9) that

$$
\prod_{j=1}^{k}\left|\lambda_{j}\left(A\left(\frac{A^{v} B^{1-v}+A^{1-v} B^{v}}{2}\right) B\right)\right| \leq \prod_{j=1}^{k} \lambda_{j}\left(\frac{A+B}{2}\right)^{3}
$$

This completes the proof.

Remark 2.3 As an application of Theorem 2.2, we now present a matrix version of (1.3). Taking $v=\frac{1}{2}$ in this last inequality, we have

$$
\prod_{j=1}^{k}\left|\lambda_{j}\left(A^{3 / 2} B^{3 / 2}\right)\right| \leq \prod_{j=1}^{k} s_{j}\left(\frac{A+B}{2}\right)^{3}
$$

and so

$$
\prod_{j=1}^{k} s_{j}\left(A^{3 / 4} B^{3 / 4}\right) \leq \prod_{j=1}^{k} s_{j}\left(\frac{A+B}{2}\right)^{3 / 2}
$$

which is equivalent to

$$
\prod_{j=1}^{k} s_{j}\left(\left|A^{3 / 4} B^{3 / 4}\right|^{2 / 3}\right) \leq \prod_{j=1}^{k} s_{j}\left(\frac{A+B}{2}\right) .
$$

Since weak log-majorization is stronger than weak majorization, we have

$$
\sum_{j=1}^{k} s_{j}\left(\left|A^{3 / 4} B^{3 / 4}\right|^{2 / 3}\right) \leq \sum_{j=1}^{k} s_{j}\left(\frac{A+B}{2}\right)
$$

By Fan's dominance theorem [4, p.93], we get

$$
\left\|\left|A^{3 / 4} B^{3 / 4}\right|^{2 / 3}\right\| \leq \frac{1}{2}\|A+B\| .
$$

This is a matrix version of (1.3). 
Next, we give another proof of the inequality (2.10). Araki [14] (also see [15]) obtained the following log-majorization inequality:

$$
\prod_{j=1}^{k} s_{j}\left(\left(A^{p / 2} B^{p} A^{p / 2}\right)^{q / p}\right) \leq \prod_{j=1}^{k} s_{j}\left(A^{q / 2} B^{q} A^{q / 2}\right), \quad 0<p \leq q .
$$

Putting

$$
p=\frac{3}{2}, \quad q=2
$$

in (2.11) gives

$$
\prod_{j=1}^{k} s_{j}\left(\left(A^{3 / 4} B^{3 / 2} A^{3 / 4}\right)^{1 / 3}\right) \leq \prod_{j=1}^{k} s_{j}\left(A B^{2} A\right)^{1 / 4}
$$

and so

$$
\sum_{j=1}^{k} s_{j}\left(\left|A^{3 / 4} B^{3 / 4}\right|^{2 / 3}\right) \leq \sum_{j=1}^{k} s_{j}\left(|A B|^{1 / 2}\right) .
$$

By Fan's dominance theorem [4, p.93], we get

$$
\left\|\left|A^{3 / 4} B^{3 / 4}\right|^{2 / 3}\right\| \leq\left\||A B|^{1 / 2}\right\| .
$$

It follows from (2.1) and (2.12) that

$$
\left\|\left|A^{3 / 4} B^{3 / 4}\right|^{2 / 3}\right\| \leq \frac{1}{2}\|A+B\| .
$$

\section{Competing interests}

The authors declare that they have no competing interests.

\section{Authors' contributions}

All authors contributed equally to the manuscript and read and approved the final manuscript.

\section{Acknowledgements}

The authors wish to express their heartfelt thanks to the referees for their detailed and helpful suggestions for revising the manuscript.

\section{Received: 5 July 2012 Accepted: 14 December 2012 Published: 4 January 2013}

\section{References}

1. Bhatia, R, Kittaneh, F: Notes on matrix arithmetic-geometric mean inequalities. Linear Algebra Appl. 308, $203-211$ (2000)

2. Drury, SW: On a question of Bhatia and Kittaneh. Linear Algebra Appl. 437, 1955-1960 (2012)

3. Audenaert, KMR: A singular value inequality for Heinz means. Linear Algebra Appl. 422, 279-283 (2007)

4. Bhatia, R: Matrix Analysis. Springer, New York (1997)

5. Bhatia, R, Davis, C: More matrix forms of the arithmetic-geometric mean inequality. SIAM J. Matrix Anal. Appl. 14, 132-136 (1993)

6. Bhatia, R, Kittaneh, F: On the singular values of a product of operators. SIAM J. Matrix Anal. Appl. 11, 272-277 (1990)

7. Bhatia, R: Interpolating the arithmetic-geometric mean inequality and its operator version. Linear Algebra Appl. 413, 355-363 (2006)

8. Bhatia, R, Kittaneh, F: The matrix arithmetic-geometric mean inequality revisited. Linear Algebra Appl. 428, 2177-2191 (2008) 
9. Kosaki, H: Arithmetic-geometric mean and related inequalities for operators. J. Funct. Anal. 156, $429-451$ (1998)

10. Bhatia, R: Positive Definite Matrices. Princeton University Press, Princeton (2007)

11. Zhan, X: Matrix Inequalities. Lecture Notes in Mathematics, vol. 1790. Springer, Berlin (2002)

12. Ando, T: Matrix Young inequalities. In: Operator Theory in Function Spaces and Banach Lattices. Oper. Theory Adv. Appl., vol. 75, pp. 33-38 (1995)

13. Tao, Y: More results on singular value inequalities of matrices. Linear Algebra Appl. 416, 724-729 (2006)

14. Araki, H: On an inequality of Lieb and Thirring. Lett. Math. Phys. 19, 167-170 (1990)

15. Hiai, F: Matrix analysis: matrix monotone functions, matrix means, and majorization. Interdiscip. Inf. Sci. 16, 139-248 (2010)

doi:10.1186/1029-242X-2013-6

Cite this article as: Xu and He: Inequalities for eigenvalues of matrices. Journal of Inequalities and Applications 2013 2013:6.

Submit your manuscript to a SpringerOpen ${ }^{\circ}$ journal and benefit from:

- Convenient online submission

- Rigorous peer review

- Immediate publication on acceptance

- Open access: articles freely available online

- High visibility within the field

- Retaining the copyright to your article 